\title{
Spontaneous Brain Activity Predicts Learning Ability of Foreign Sounds
}

\author{
Noelia Ventura-Campos, ${ }^{1}$ Ana Sanjuán, ${ }^{1}$ Julio González, ${ }^{1}$ María-Ángeles Palomar-García, ${ }^{1}$ Aina Rodríguez-Pujadas, ${ }^{1}$ \\ Núria Sebastián-Gallés, ${ }^{2}$ Gustavo Deco, ${ }^{3,4}$ and César Ávila ${ }^{1}$ \\ ${ }^{1}$ Departament de Psicologia Bàsica, Clínica i Psicobiología, Universitat Jaume I, 12071 Castellón de la Plana, Spain, ${ }^{2}$ Center for Brain and Cognition, \\ Department of Technologies and ${ }^{3}$ Theoretical and Computational Neuroscience Group, Universitat Pompeu Fabra, 08018 Barcelona, Spain, and ${ }^{4}$ Institució \\ Catalana de Recerca i Estudis Avançats, 08010 Barcelona, Spain
}

Can learning capacity of the human brain be predicted from initial spontaneous functional connectivity (FC) between brain areas involved in a task? We combined task-related functional magnetic resonance imaging (fMRI) and resting-state fMRI (rs-fMRI) before and after training with a Hindi dental-retroflex nonnative contrast. Previous fMRI results were replicated, demonstrating that this learning recruited the left insula/frontal operculum and the left superior parietal lobe, among other areas of the brain. Crucially, resting-state FC (rs-FC) between these two areas at pretraining predicted individual differences in learning outcomes after distributed (Experiment 1) and intensive training (Experiment 2). Furthermore, this rs-FC was reduced at posttraining, a change that may also account for learning. Finally, resting-state network analyses showed that the mechanism underlying this reduction of rs-FC was mainly a transfer in intrinsic activity of the left frontal operculum/anterior insula from the left frontoparietal network to the salience network. Thus, rs-FC may contribute to predict learning ability and to understand how learning modifies the functioning of the brain. The discovery of this correspondence between initial spontaneous brain activity in task-related areas and posttraining performance opens new avenues to find predictors of learning capacities in the brain using task-related fMRI and rs-fMRI combined.

\section{Introduction}

People vary in their ability to learn new skills. Dating back to phrenology, neuroscientists have attempted to understand where these different learning capacities originate by exploring the brain. The effect of learning on the brain has been indexed through diverse techniques such as functional magnetic resonance imaging (fMRI) and morphometry (Lewis et al., 2009; Takeuchi et al., 2011). More recently, resting-state fMRI (rsfMRI) has become another possible candidate to predict learning capacities (Baldassarre et al., 2012). Rs-fMRI gives an intrinsic and coherent signal within a number of replicable networks with topography closely resembling that of functional networks recruited during tasks (Smith et al., 2009). Importantly, previous studies demonstrated that resting-state functional connectivity (rs-FC) changes during rs-fMRI occur in parallel with fMRI

Received Oct. 1, 2012; revised April 9, 2013; accepted April 16, 2013.

Author contributions: N.V.-C., A.S., J.G., N.S.-G., G.D., and C.A. designed research; N.V.-C., A.S., J.G., M.A.P.-G., A.R.-P., N.S.-G., G.D., and C.A. performed research; N.V.-C. and C.A. contributed unpublished reagents/analytic tools; N.V.-C., M.A.P.-G., and C.A. analyzed data; N.V.-C., N.S.-G., G.D., and C.A. wrote the paper.

This work was supported in part by Spanish Ministerio de Ciencia e Innovación Grants PSI2010-20168, PSI201234071, and CONSOLIDER-INGENI0 2010 Programme CDS-2007-00012, Generalitat Valenciana Grant APOSTD/ 2012068, and Universitat Jaume I Grant P1-1B2012-38. G.D. was supported by the ERC Advanced Grant DYSTRUCTURE (no. 295129). by the Spanish Research Project SAF2010-16085, and by the CONSOLIDER-INGENI0 2010 Program CSD2007-00012.

The authors declare no competing financial interests.

Correspondence should be addressed to Noelia Ventura-Campos or César Ávila, Departamento de Psicología Básica, Clínica i Psicobiología, Universitat Jaume I, Edificio de Investigación II, Avenida Sos Baynat, s/n, C.P. 12071, Castelló de la Plana, Spain. E-mail: venturan@uji.es.

DOI:10.1523/JNEUROSCI.4655-12.2013

Copyright $\odot 2013$ the authors $\quad 0270-6474 / 13 / 339295-11 \$ 15.00 / 0$ learning-related changes (Lewis et al., 2009), and that pretraining rs-FC between visual areas of the brain predicts long-term visuomotor learning (Baldassarre et al., 2012). These contributions lay the groundwork for the possibility of using rs-fMRI guided by task-related fMRI as an index of the brain's ability to learn. The aforementioned study (Baldassarre et al., 2012), however, did not directly test this possibility due to differences in baseline performance and because the brain areas that change in rs-FC due to learning were not the same as those that predict performance at pretraining.

Our objective is to extend those results and test a more generalizable method for studying the brain's capacity to learn by determining FC during rs-fMRI between task-related brain areas. To this aim, we focused on the ability to distinguish a difficult nonnative phonetic contrast, a task at which adults typically demonstrate at-chance baseline performance but may considerably improve on with phonetic training (Golestani and Zatorre, 2009). Phonetic learning has been consistently linked to activity of the left frontal operculum/anterior insula (LFO/aI) in association with different temporal and parietal areas (Golestani and Zatorre, 2004; Deng et al., 2008), and with increased gray and white matter in the left inferior and superior parietal gyri (Golestani et al., 2002; Golestani and Zatorre, 2004). In Experiment 1, we examined the effect of distributed phonetic learning on the brain based on changes in the blood oxygen level-dependent (BOLD) signal recorded both during the task and at rest. Participants were scanned using resting-state and task-related fMRI before and after 2 weeks of phonetic training to discriminate new phonemes. Task-related fMRI was used to identify brain areas 


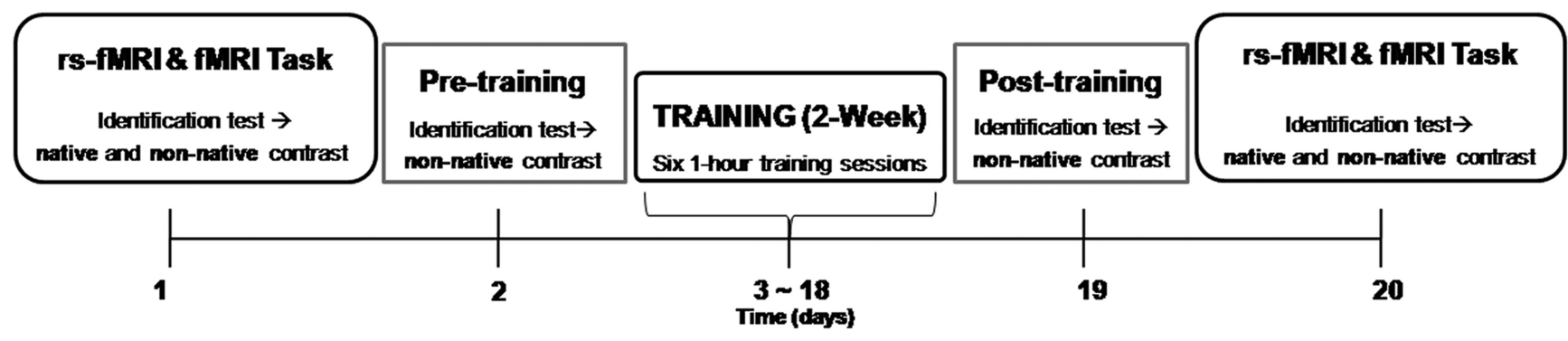

Figure 1. Schematic time representation of the experimental procedure. The first fMRI data acquisition was followed by the pretraining test, training sessions (2 week behavioral phonetic training with the nonnative contrast), posttraining test, and second fMRI data acquisition.

involved in phonetic discrimination, whereas rs-fMRI was used to calculate rs-FC between these task-related areas at pretraining and posttraining. Experiment 2 was designed to confirm the most relevant result of Experiment 1 (the significant correlation between pretraining rs-fMRI and learning) in a larger sample and using a single day of intensive training for a mean of $75 \mathrm{~min}$.

\section{Materials and Methods Experiment 1 \\ Participants}

Twenty-two right-handed participants were initially recruited for this study. Three participants were excluded from analyses because they did not respond to over $51 \%$ of the nonnative phonetic contrast trials in posttraining [following the logic of Golestani and Zatorre (2004)]. The final sample consisted of nineteen participants (mean age, 23.74 years; SD, 2.54; nine males) with normal auditory acuity. None of the participants had previous experience with languages using retroflex phonemes. All participants gave informed consent before participation and received monetary compensation for their time and effort. This research was approved by the Universitat Jaume I Ethics Committee.

\section{Task and training data}

Experimental overview. The procedure was similar to that implemented by Golestani and Zatorre (2004). Imaging data consisted of an rs-fMRI followed by a phoneme identification fMRI task performed before and after 2 weeks of behavioral phonetic training with the nonnative phoneme contrast (Fig. 1).

Stimuli. We selected the dental-retroflex place-of-articulation contrast, which is used in languages of India such as Hindi and Urdu. Retroflex consonants require a relatively complex articulation. They are rare across the languages of the world and crucially, they are not used phonemically in Spanish. Perceptually, Spanish listeners assimilate the dentalretroflex sounds such that they perceive both sounds as instances of the dental consonant/d/. Previous research has shown that this contrast is difficult to perceive for listeners of languages without the dental-retroflex contrast (such as French and English) (Werker and Lalonde, 1988; Polka, 1991; Golestani and Zatorre, 2004).

Our nonnative phonetic stimuli were the same as those used by Golestani and Zatorre $(2004,2009)$. There were seven stimuli varying in equal steps in terms of acoustic difference between adjacent items: Stimulus 1 corresponded to the dental/da/, and Stimulus 7 to the retroflex voiced/da/, a prototype of the unaspirated stop consonant. The stimuli can be listened to at the following website: http://www. zlab.mcgill.ca/supplements/language-anatomy.html.

Stimuli for the phoneme identification fMRI task. Apart from the two end point sounds (i.e., Stimulus 1 and Stimulus 7) corresponding to the nonnative phoneme contrast, two control stimuli were used in the fMRI task. The first was a native phoneme contrast that constituted two stop dental sounds: a voiced/da/ sound similar to Stimulus 1 (above) and a voiceless/ta/ sound. As this contrast is used in the Spanish language, it is easy for native Spanish listeners to distinguish. Both sounds were synthesized (Klatt, 1980) using parameters based on Golestani and Zatorre's study $(2004,2009)$. The second control stimulus was a burst of white noise matched in sound pressure level and duration with the consonant- vowel stimuli. It was digitized at a sampling rate of $22.050 \mathrm{~Hz}$ using a 16 bit analog-to-digital converter and low-pass filtered at $11.025 \mathrm{~Hz}$.

Testing and behavioral training. The testing and the behavioral training task were implemented using Inquisit by Millisecond software (http://www.millisecond.com).

For behavioral training, participants underwent six $1 \mathrm{~h}$ behavioral training sessions spread out over the course of 2 weeks. During the sessions, participants were instructed to identify the nonnative phoneme contrast: dental versus retroflex sounds. Training involved 20-trial blocks of the identification task, after each trial participants received feedback on the accuracy of their response. We implemented the fading technique during training: participants began by identifying the pair of end point tokens of a synthetic continuum; then, depending on performance, the acoustic differences between the sounds were progressively reduced until the phonetic differences were near the categorical boundary. Training was discontinued once a participant achieved criterion on this last contrast (at least $80 \%$ correct responses) or had completed a maximum of 200 trials (10 blocks) (Golestani and Zatorre, 2009). The fading technique is aimed at helping the listener attend to the relevant phonetic/acoustic properties of category distinctions, beginning by presenting the most easily perceived phonetic/acoustic differences and ending with phonetic/acoustic differences near the categorical boundary. Within each session, participants completed this training twice, separated by $10 \mathrm{~min}$ of rest, spending an average of $60 \mathrm{~min}$ per day (range, 50-70 $\mathrm{min})$.

The pretraining and posttraining identification test. Before and after training, participants performed a behavioral identification task without feedback on their performance. They heard 20 randomly presented instances of each of the two end point stimuli and were required to press the button corresponding to the type of voicing, dental or retroflex. The overall percentage of correct responses was used as a behavioral measure of nonnative identification outside the scanner.

Procedure for the phoneme identification fMRI task. All participants performed the phoneme identification task with protocol similar to that of previous studies (Golestani and Zatorre, 2004): Participants were familiarized with the stimuli and underwent a short practice task before entering the scanner. The task was programmed in presentation software (Neurobehavioral Systems), and the stimuli were presented through headphones compatible with MRI (VisuaStim; Resonance Technology). We used a sparse-sampling design to mitigate the interference of scanner noise by inserting a $2 \mathrm{~s}$ delay between image acquisitions (Staeren et al., 2009). Stimuli were presented during the silent periods of each $3.6 \mathrm{~s}$ TR period with different onsets $(1.9,2.1$, and $2.3 \mathrm{~s})$. The three different conditions (native, nonnative, and noise burst) were presented in a block design (six stimuli; block duration, $21.6 \mathrm{~s}$ ). Each block was followed by silent periods of 3.6 or $7.2 \mathrm{~s}$. The order of the conditions was counterbalanced.

Responses were collected during fMRI scanning for all conditions. Participants were instructed to stay attentive, listen to each sound, and push one of the two buttons of the ResponseGrip (Nordic NeuroLab) with their right hand: one button if they heard a native or nonnative dental sound/da/, and the other button if they heard a native dental sound/ta/ or nonnative retroflex sound/da/. For the noise burst condition, participants were asked to randomly press either of the two response 
buttons. Data from one participant were lost due to technical problems during the task. The overall percentage of correct responses in native and nonnative blocks was used as a behavioral measure of performance inside the scanner.

\section{Image data acquisition}

The same fMRI protocol was used before and after training. fMRI sessions consisted of a resting state in which participants were instructed to simply rest with their eyes closed and not to sleep or think about anything in particular. This was followed by the phoneme identification fMRI task in which participants remained with their eyes closed. Images were acquired on a 1.5 T scanner (Siemens Symphony). Participants were placed in a supine position in the MRI scanner, and their heads were immobilized with cushions to reduce motion artifacts. For the rs-fMRI, a total of 270 volumes were recorded over $9 \mathrm{~min}$ using a gradient-echo $\mathrm{T}^{*}$ weighted echoplanar imaging sequence (TR, $2000 \mathrm{~ms}$; TE, $48 \mathrm{~ms}$; matrix, $64 \times 64$; voxel size, $3.5 \times 3.5 \mathrm{~mm}$; flip angle, $90^{\circ}$; slice thickness, $4 \mathrm{~mm}$; slice gap, $0.8 \mathrm{~mm}$ ). We acquired 24 interleaved axial slices parallel to the anterior-posterior commissure plane covering the entire brain. For the fMRI task, a total of 293 volumes were sparsely acquired over 17:58 min using a gradient-echo $\mathrm{T} 2^{*}$-weighted echoplanar imaging sequence (TR, $3600 \mathrm{~ms}$; TA, $1600 \mathrm{~ms}$; TE, $46 \mathrm{~ms}$; matrix, $64 \times 64$; voxel size, $3.5 \times 3.5$ $\mathrm{mm}$; flip angle, $90^{\circ}$; slice thickness, $4 \mathrm{~mm}$; slice gap, $0.4 \mathrm{~mm}$ ). We acquired 19 interleaved axial slices in the orientation of the Sylvian fissure. Before the functional magnetic resonance sequences, a high-resolution structural T1-weighted MPRAGE sequence was acquired (TR, $2200 \mathrm{~ms}$; TE, $3.8 \mathrm{~ms}$; matrix, $256 \times 256 \times 160$; voxel size, $1 \times 1 \times 1 \mathrm{~mm}$ ).

\section{FMRI task}

Phoneme identification $f M R I$ task analysis. Image preprocessing using SPM8 software (Wellcome Trust Centre for Neuroimaging, London, UK) consisted of head motion correction, spatial normalization $\left(3 \mathrm{~mm}^{3}\right)$ to the Montreal Neurological Institute (MNI) space, and spatial smoothing by convolution with an isotropic Gaussian kernel of $8 \mathrm{~mm}$ full width at half maximum (FWHM). In the first-level analyses, a general linear model was performed for each participant and for each time period (before and after training), modeling the conditions of interest corresponding to native, nonnative, noise, and silence using a boxcar function. The parameters of movement correction were used as covariates of no interest. Temporal autocorrelation was not applied to the sparsesampling model in the first-level single-subject analysis. To assess the effects of native and nonnative conditions, we performed two comparisons of interest to produce a "contrast image" for each participant: native versus noise (native contrast) and nonnative versus noise (nonnative contrast). In a random-level analysis, we used a one-sample $t$ test for each contrast image to obtain population inferences for each condition, native and nonnative, in each period. To estimate the changes between pretraining and posttraining, we used a paired $t$ test design. We also performed a multiple regression analysis on the learning measure (posttraining minus pretraining identification scores) and the BOLD signal during identification of the nonnative contrast in posttraining. All results were thresholded at $p<0.05$, familywise error (FWE) corrected for multiple comparisons at the cluster level determined by whole-brain Monte Carlo simulations using the AlphaSim program in REST software (http://www. restfmri.net; voxelwise threshold of $p<0.005$ and cluster-size criterion of 42 voxels).

Post hoc definition of ROIs used as seed regions in the rs-fMRI analysis. Following a similar approach to that of previous reports (Lewis et al., 2009), the seed regions selected for the rs-fMRI analysis were extracted from the phoneme identification fMRI task by comparing the trained (i.e., nonnative contrast) and untrained (i.e., native contrast) conditions. Both conditions were similar in task instructions and behavioral results. We defined two different contrasts: (1) To identify the functional regions specifically associated with the effects of training on Hindi phonemic identification, we computed the contrast Posttraining Nonnative $>$ Pretraining Native using an inclusive mask of the posttraining nonnative contrast $(p<0.05$, uncorrected at the voxel level). This inclusive mask restricted analysis only to voxels found in areas linked to the nonnative condition after training. (2) To identify the functional regions specifi- cally associated with the effects of processing the native contrast, we computed the contrast Pretraining Native $>$ Posttraining Nonnative using an inclusive mask of the pretraining native contrast ( $p<0.05$, uncorrected at the voxel level). This inclusive mask restricted the analysis to voxels found in areas linked to the native condition before training that did not participate in nonnative processing. These results were thresholded at $p<0.05$, FWE corrected for multiple comparisons at the cluster level determined by whole-brain Monte Carlo simulations. Choosing seed regions may bias connectivity findings toward specific, smaller, or overlapping subsystems rather than larger, distinct networks (Buckner et al., 2008). Therefore, the location of each seed region must be a "good" representative of the set of correlated voxels at rest, and it is important to consider both seed size and location (Beckmann et al., 2005; Cole et al., 2010). For this reason, we applied a more restrictive voxelwise threshold of $p<0.001$ using a cluster-size criterion of 21 to obtain the location of peak $z$ scores that were more highly significant. Then, the seed regions were functionally defined as 8-mm-radius spheres centered on peaks of local maxima obtained by previous contrasts of the task-related fMRI; peaks within $8 \mathrm{~mm}$ of each other were consolidated into a single seed (Lewis et al., 2009).

\section{rs-FC analyses}

Preprocessing. Rs-fMRI datasets were processed using a toolkit of the Data Processing Assistant for Resting-State fMRI (DPARSF; http://www. restfmri.net) (Chao-Gan and Yu-Feng, 2010). The rs-fMRI preprocessing included the slice-timing correction for interleaved acquisitions using sinc interpolation and resampling with the middle slice (23rd) in time as the reference point, head motion correction, spatial normalization to the MNI space $\left(3 \mathrm{~mm}^{3}\right)$, and spatial smoothing with an isotropic Gaussian kernel of $4 \mathrm{~mm}$ FWHM.

Additional preprocessing for seed-based rs-FC analysis. We conducted additional preprocessing through the following steps: (1) removing the linear trend in the time series, (2) temporally bandpass filtering $(0.01-$ $0.08 \mathrm{~Hz}$ ) to reduce the effect of low-frequency drift and high-frequency noise (Biswal et al., 1995; Lowe et al., 1998), and (3) controlling the nonneural noise in the seed region time series (Fox et al., 2005). Several sources of spurious variance were removed from the data through linear regression: six parameters from rigid body correction of head motion, the global mean signal, the white matter signal, and the CSF signal.

Seed-based rs-FC analyses. After the preprocessing of rs-fMRI data, we used the predefined seed regions for ROI-wise rs-FC analyses using the DPARSF toolbox. The mean time course of all voxels in each seed region was used to calculate pairwise linear correlations (Pearson's correlation) during each rs-fMRI period. Individuals' $r$ values were normalized to $z$ values using Fisher's $z$ transformation. To examine the changes in rs-FC before and after training, we performed a paired $t$ test on the $z$ value for each rs-fMRI period using the Statistical Package for the Social Sciences (SPSS), version 19.0. The multiple comparison of pairwise correlation analysis threshold was set to $p<0.05$, applying a Bonferroni correction. Based on this method, we divided the a priori selected threshold of $p<0.05$ by the number of tests performed ( $k=6$; see Results), which stabilized statistical levels as significant if $p<0.0083$. We also performed Spearman's correlation analysis between the values of the identification test and (1) the rs-FC of each seed region in pretraining, (2) the rs-FC of each seed region in posttraining, and (3) the changes in rs-FC (posttraining minus pretraining).

Intrinsic rs-FC network analyses. Spontaneous activity measured with rs-fMRI is organized in a limited number of brain networks, and this finding has been replicable across studies (Damoiseaux et al., 2006; Shehzad et al., 2009). Three steps were completed to identify the resting-state networks (RSNs) evoked by the seed regions (Seeley et al., 2007). We performed an additional fourth step to investigate how phonetic learning modified the intrinsic rs-FC of these brain networks.

Step 1: Independent component analysis data. We performed independent component (IC) analyses (ICAs) using the Group ICA of fMRI Toolbox (GIFT; http://icatb.sourceforge.net/groupica.htm) (Calhoun et al., 2001) for each rs-fMRI period to obtain the known RSNs with a convergent method in a large dataset (Beckmann et al., 2005; Damoiseaux et al., 2006). Previous studies have demonstrated that a high model 
order ICA produces a refined IC associated with known anatomical and functional segmentation (Kiviniemi et al., 2009; Smith et al., 2009; Abou-Elseoud et al., 2010; Ystad et al., 2010; Allen et al., 2011); therefore, 40 ICs were selected for each rs-fMRI time period to obtain the most networks. At this point, we conducted group-level spatial ICA using the Infomax ICA algorithm (Bell and Sejnowski, 1995). Twenty iterations of ICA were performed using ICASSO (http://www.cis.hut.fi/projects/ica/ icasso) to determine the reliability or stability of the ICA algorithm (Himberg et al., 2004), and the best estimate (centrotype of the cluster) for each IC was used. The individual IC maps and time courses were computed using backreconstruction based on aggregate components of the ICA and the results from the data reduction step (Calhoun et al., 2001, 2002; Erhardt et al., 2011). The RSNs were classified by visually inspecting the aggregate spatial maps (discarding the ICs associated with physiological artifacts) and average power spectra $(<0.10 \mathrm{~Hz})$. (Lowe et al., 1998).

Step 2: Seed-based FC map. We used the DPARSF toolbox to compute voxelwise rs-FC maps to disentangle the networks evoked by seed regions. This method allowed us to study the rs-FC (Pearson's correlation) of the seed region with all other voxels in the whole brain for each participant during each rs-fMRI period. Individual $r$ maps were normalized to $z$ maps using Fisher's $z$ transformation. For each seed region, a one-sample $t$ test using SPM8 was performed by entering the $z$ maps to detect brain areas showing significant rs-FC across participants and obtain FC maps in pretraining and posttraining $(p<0.05$, FWE corrected for multiple comparisons at the cluster level determined by whole-brain Monte Carlo simulation with voxelwise threshold of $p<0.005$ and a cluster-size criterion of 12 voxels).

Step 3: Selection of RSNs associated with each seed. To select the RSN obtained by the ICA that best fit the FC map of each seed region, we used the spatial correlation sorting option in GIFT to examine the spatial correlation among all the ICs obtained in Step 1 and the FC maps obtained in Step 2 independently for pretraining and posttraining rs-fMRI. Then, we selected the ICs that demonstrated the highest correlations with each FC map and confirmed this through visual evaluation (Correa et al., 2007). This approach allowed us to determine the IC for each participant that showed greater spatial correlation with each FC map, and this IC was selected as the RSN associated with the seed corresponding to the FC map.

Step 4: Intrinsic rs-FC of seeds within RSNs. In the last step, a randomeffects statistical analysis was performed for each RSN selected in Step 3 before and after training using a one-sample $t$ test (threshold $z$ score $>3$ ) to obtain the population inferences for each RSN. To calculate the intrinsic rs-FC of each seed, we extracted the first eigenvariate of the voxels of the seed corresponding to each selected RSN across participants using VOI command of SPM8. In SPM8, the first eigenvariate is the estimated weighted mean of the VOI (Friston et al., 2006). Also, a paired $t$ test was computed using SPSS to evaluate the difference in mean intrinsic rs-FC of each seed within each RSN before and after training. The multiple comparison threshold was set to $p<0.05$, and a Bonferroni correction was applied. Based on this method, we divided the a priori selected threshold of $p<0.05$ by the number of tests performed $(k=3$; see Results), which stabilized statistical levels as significant if $p<0.016$. Finally, we performed a correlation analysis between the changes in intrinsic rs-FC within the RSN (only those for which the paired $t$ test was significant) and the posttraining rs-FC obtained by seed-based rs-FC analyses. This correlation analysis was performed to investigate whether the changes in rs-FC between seed regions due to training were related to the intrinsic rs-FC within the brain networks.

\section{Experiment 2}

Participants

Twenty-eight new participants were recruited for this study (mean age, 22.18 years; SD, 3.62; 12 males). Inclusion and exclusion criteria were the same as in Experiment 1. No participant was discarded in this experiment.

\section{Testing and behavioral training}

Outside the scanner, participants were tested three times and trained intensively for $60-80 \mathrm{~min}$ in a single day. We used only the two end point stimuli corresponding to the dental and retroflex sounds. The behavioral task was divided into three identical blocks that consisted of 200 trials of behavioral identification training with feedback followed by 100 trials of an identification test without feedback (Tests 1,2, and 3); these blocks were separated by $10 \mathrm{~min}$ rest periods. Performance was measured using the mean percentage of correct responses during each identification test.

\section{Imaging data acquisition}

The fMRI session consisted of a single resting state acquired before training on a $1.5 \mathrm{~T}$ scanner (Siemens Avanto). A total of 270 volumes were recorded over $9 \mathrm{~min}$ using a gradient-echo $\mathrm{T} 2^{\star}$-weighted echoplanar 
imaging sequence (TR, $2000 \mathrm{~ms}$; TE, $30 \mathrm{~ms}$; matrix, $64 \times 64 \times 30$; voxel size, $3.5 \times 3.5 \times 4.02 \mathrm{~mm}$; flip angle, $\left.90^{\circ}\right)$. Before the functional sequences, a T1-weighted sequence was acquired (TR, $11 \mathrm{~ms}$; TE, $4.9 \mathrm{~ms}$; FOV, $24 \mathrm{~cm}$; matrix, $256 \times 224 \times 176$; voxel size, $1 \times 1 \times 1 \mathrm{~mm}$ ).

Seed-based rs-FC analysis

The preprocessing and the seed-based rs-FC analyses were as in Experiment 1. For the rs-FC analysis, we selected the same seed regions related to learning as in Experiment 1 (for details, see Experiment 1). To replicate the results obtained in Experiment 1 regarding the ability of initial rs-FC to predict learning ability, we performed a Spearman's correlation analysis between the pretraining rs-FC and posttraining performance (Test 3). Finally, partial correlation analyses controlling for Tests 1 and 2 were performed to investigate learning effects.

\section{Results}

\section{Experiment 1}

Behavioral data

Behavioral results showed at chance accuracy at baseline and a significant increased mean accuracy after learning (Fig. 2). Performance inside and outside the scanner did not correlate before training $(p>0.10)$ but were strongly correlated after training $(r=0.55, p<0.001, n=18)$. Moreover, there were no significant correlations between pretraining and posttraining performance for the nonnative contrast (inside scanner, $r=0.16, p<0.51, n=$ 18 ; outside scanner, $r=0.005, p<0.98, n=19)$, but there was a trend toward significant correlation between both measures for the native contrast (inside scanner, $r=0.44, p<0.07, n=18$ ).

We analyzed performance inside the scanner using a two by two repeated-measures ANOVA with contrast (native/nonnative) and time (pre/post) as within-subjects factors. The analysis yielded significant main effects for contrast $\left(F_{(1,17)}=495.81, p<\right.$ $0.001)$ and time $\left(F_{(1,17)}=18.64, p<0.001\right)$. As expected, the contrast by time interaction reached significance $\left(F_{(1,17)}=5.14\right.$, $p<0.04)$, indicating more learning for the nonnative contrast than for the native contrast. Multivariate ANOVAs for each contrast separately showed better performance for the nonnative contrast after learning $\left(F_{(1,17)}=16.92, p<0.04\right)$, but not for the native contrast $(p>0.06)$. Performance outside the scanner was analyzed using a paired $t$ test that revealed a significant increment of performance after learning $\left(t_{(18)}=9.38, p<0.001\right)$.

To discard the possibility that neural differences were driven by performance confounds such as different response speeds (Poldrack, 2000), we collected response times inside the scanner before and after training. The response time (in seconds) of the native condition (pretraining, mean, $0.69 ; \mathrm{SD}, 0.11$; posttraining, mean, 0.65 ; SD, $0.14 ; n=18$ ) and nonnative condition (pretraining, mean, 0.77; SD, 0.13; posttraining, mean, 0.85 ; SD, 0.19 ; $n=18$ ) did not differ between both time periods, removing this potential confound $(p>0.10)$. Thus, differences in brain activity between pretraining and posttraining were not due to differences in response speed.

\section{The phoneme identification $f M R I$ task}

One-sample $t$ tests yielded results similar to those of a previous study (Golestani and Zatorre, 2004). We obtained significant activations for the native contrast (native minus noise) during pretraining in the bilateral inferior frontal operculum/anterior insula regions, bilateral superior temporal gyrus, right middle frontal gyrus, and right inferior parietal lobe (Fig. 3A). The same contrast after training involved similar regions with additional activation in the left inferior parietal lobe and bilateral caudate head (Fig. 3B). For the nonnative contrast (nonnative minus noise), significant activations were obtained in the bilateral inferior frontal operculum/anterior insula regions, bilateral superior
A
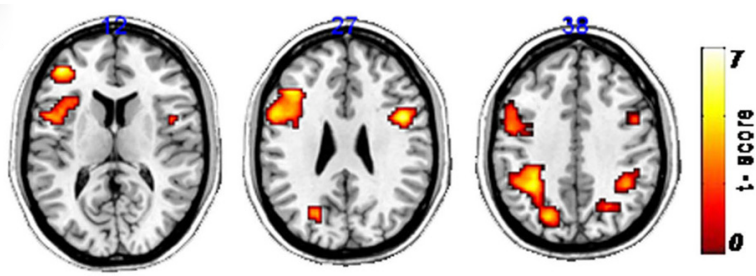

B
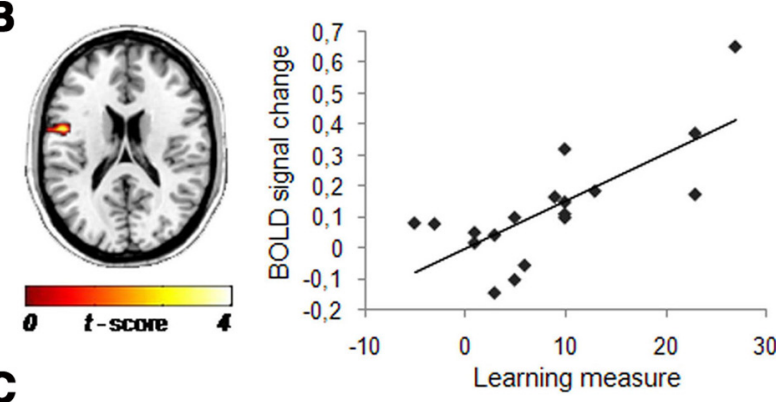

C

$L$
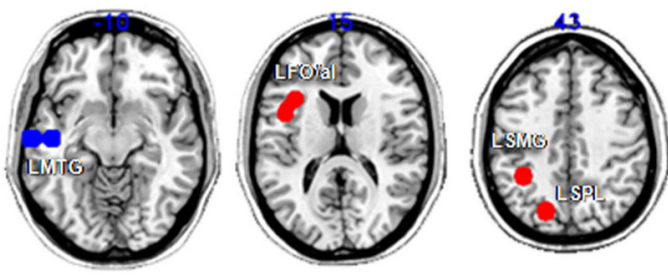

$\boldsymbol{R}$

Figure 4. Results of the phoneme identification fMRI task. $A$, Posttraining versus pretraining nonnative contrast: statistical parametric maps representing the comparison of posttraining versus pretraining brain activity during the nonnative contrast (nonnative minus noise) corrected for multiple comparisons (FWE correction at $p<0.05$ determined by Monte Carlo simulation). Similar to the results of Golestani and Zatorre (2004), the effects of nonnative contrast training resulted in increased functional activity in the bilateral F0/al, LSPL, and LSMG. There were no significant differences in the comparison of posttraining and pretraining for the native contrast. $\boldsymbol{B}$, Performance correlates of fMRI data: the left inferior frontal gyrus (BA 44 at MNI coordinates $-54,3,27 ; z$ value, 3.51 ) was positively correlated with the learning measure (posttraining minus pretraining identification scores) recorded inside the scanner during the presentation of the posttraining nonnative contrast ( $p<0.05$, FWE corrected at the cluster level). C, ROI definition: Illustration of the seed regions selected for the rs- $\mathrm{FC}$ analysis obtained by spheres of $8 \mathrm{~mm}$ radius centered on peaks of the ROls derived from task-related fMRI data. This procedure lets us identify the brain areas involved in (1) processing of the nonnative contrast, Posttraining Nonnative > Pretraining Native (LF0/al, LSPL, and LSMG; red) and (2) processing of the native contrast, Pretraining Native $>$ Posttraining Nonnative (LMTG; blue). L, Left; $R$, right.

temporal gyrus, and right middle frontal gyrus (Fig. 3C). After training, we observed additional activations in the bilateral inferior parietal lobe, bilateral caudate head and right cingulate gyrus (Fig. 3D).

As expected, the comparison between pretraining and posttraining brain activity showed no differences for the native contrast but an increased response of the bilateral inferior frontal operculum/anterior insula region, left inferior parietal lobe and left superior parietal lobe (LSPL) after training for the nonnative contrast (Fig. 4A) (for previous results with the same task, see Golestani and Zatorre, 2004, their Table 4). Importantly, the BOLD response at the left inferior frontal gyrus (operculum) after training correlated positively with behavioral improvement (Fig. 4B).

\section{rs-FC results}

Seed-based rs-FC. ROIs derived from the fMRI task results were used as seed regions for rs-fMRI analyses (Fig. 4C). Using the contrast and mask detailed in Materials and Methods (see Post hoc definition of ROIs used as seed regions in the rs-fMRI anal- 
A $\square$ RESTpre $\square$ RESTpost
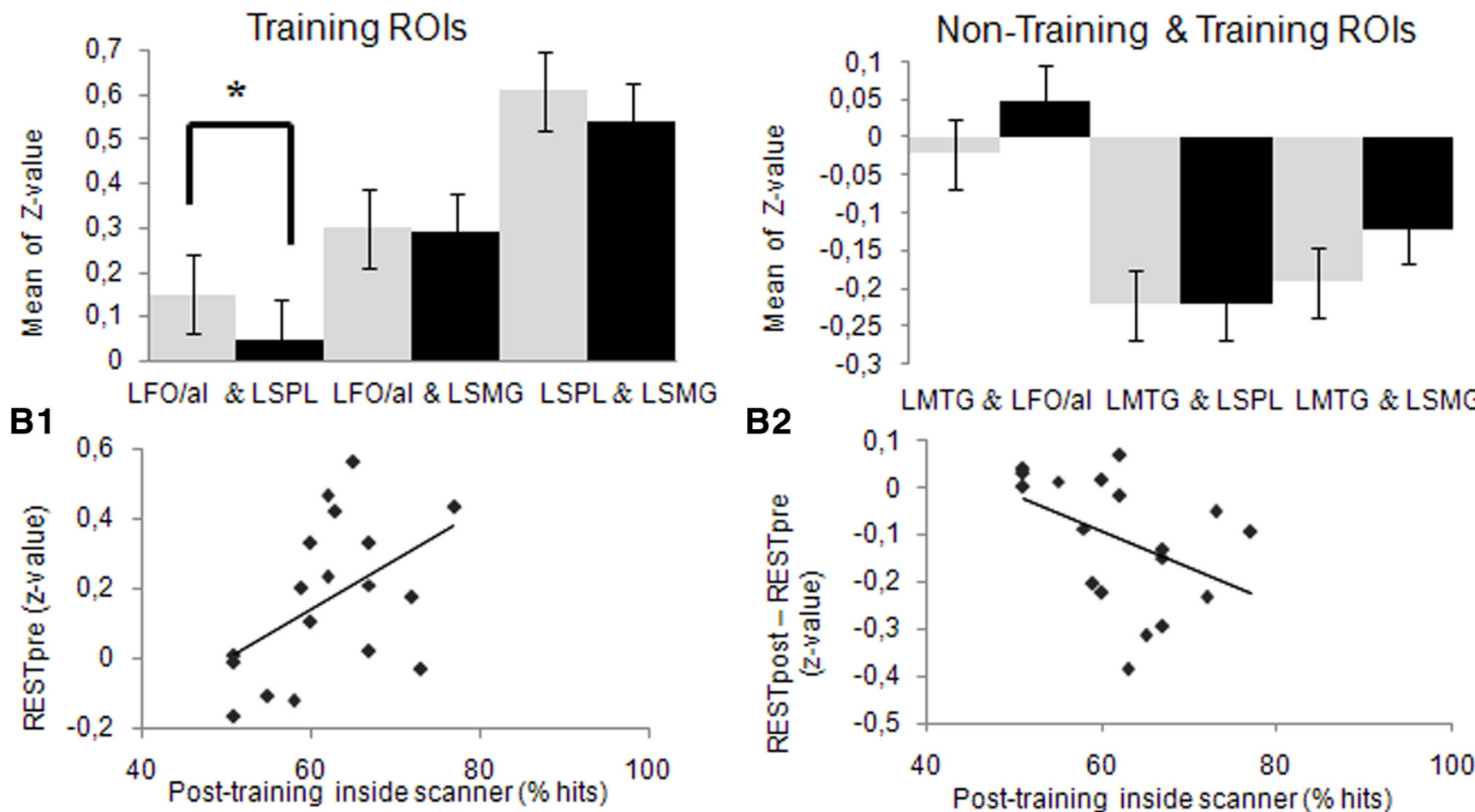

B2

LMTG \& LFO/al LMTG \& LSPL LMTG \& LSMG
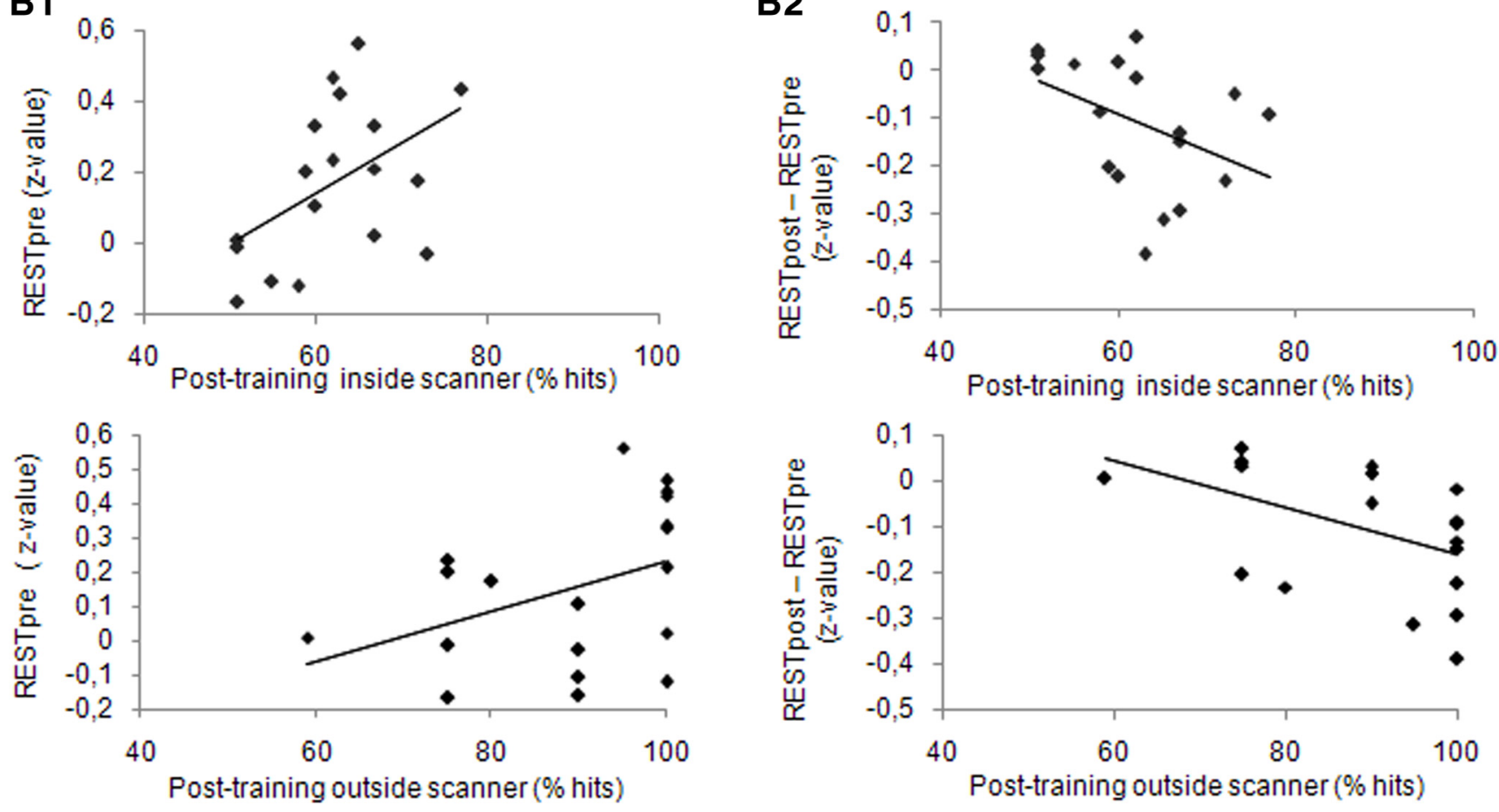

Figure 5. Changes in rs-FC associated with nonnative phoneme identification training in Experiment 1. A, Comparison of pairwise correlation coefficients ( $z$ value) of "training ROls" (LF0/al, LSMG, and LSPL) and a "nontraining ROI" (LMTG) between rs-fMRI periods [light blue, pretraining (RESTpre); dark blue, posttraining (RESTpost)]. We only observed a significant decrease in rs-FC of the LF0/al and LSPL $\left(t_{(18)}=3.27, p<0.004\right)$. B1, Pretraining rs-FC between the LF0/al and LSPL became significantly correlated with posttraining identification performance recorded inside the scanner $\left(r_{s}=0.51, p<0.05, n=18\right)$ and outside the scanner $\left(r_{s}=0.46, p<0.05, n=19\right)$. B2, Changes in rs-FC between the LF0/al and LSPL were inversely correlated with posttraining identification performance recorded inside the scanner $\left(r_{s}=-0.53, p<0.05, n=18\right)$ and outside the scanner $\left(r_{s}=-0.56, p<0.05, n=19\right)$.

ysis), we identified three seed regions associated with the effects of training (Posttraining Nonnative $>$ Pretraining Native): LFO/ aI, LSPL, and left supramarginal gyrus (LSMG). We also obtained one seed region associated with the effects of the contrast Pretraining Native $>$ Posttraining Nonnative as a control: the left middle temporal gyrus (LMTG).

To examine the rs-FC between the seed regions, we performed pairwise linear correlation analyses before and after training (Fig. $5 A$, mean $z$ values). Seed regions associated with the effects of training correlated positively between each other and negatively with the LMTG. The only significant change in rs-FC after training was a reduction of rs-FC between the LFO/aI and LSPL ( $t=$ 3.27, $p<0.004$; Fig. 5A). Critically, posttraining performance correlated positively with pretraining rs-FC between these two areas (showing that the greater the rs-FC before training, the better the learning) (Fig. 5B1), and negatively with the change (posttraining minus pretraining) in rs-FC between these areas (showing that the greater the reduction in rs-FC after training, the better the performance outcome) (Fig. 5B2).

Intrinsic rs-FC networks. To understand the reduction in the magnitude of correlation between the LFO/aI and LSPL after learning (Fig. 5A), four steps were undertaken to identify the RSNs evoked by the seed regions. First, we determined the RSNs associated with these seed regions before and after training. Following the four steps explained in Materials and Methods, we obtained the RSNs of intrinsic connectivity through ICA, identifying 14 RSNs common to both rs-fMRI periods (Fig. 6). We classified our 14 RSNs based on networks reported in previous studies (Beckmann et al., 2005; Calhoun et al., 2008; Veer et al., 2010; Allen et al., 2011), those showing that RSNs were consistent across participants and over time (Damoiseaux et al., 2006; Shehzad et al., 2009), and those demonstrating a remarkable overlap with patterns of task-induced activity (Smith et al., 2009). Then, we generated the seed-based FC maps for each seed region that 


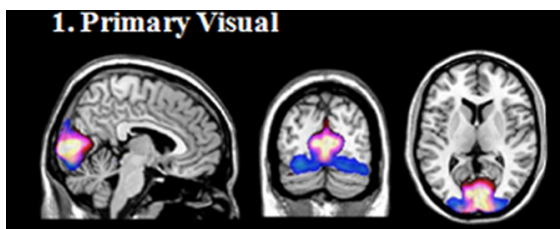

2. Lateral Visual
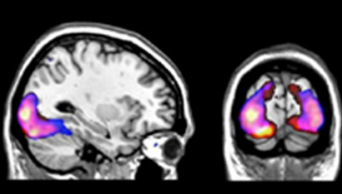

3. Medial Visual

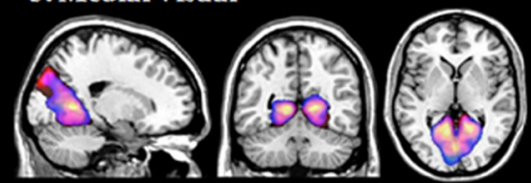

5. Precuneus

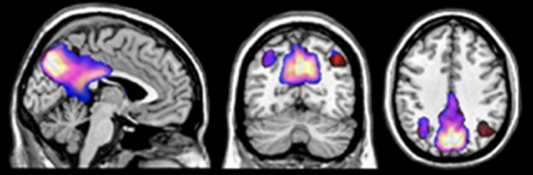

7. Sensory network (SN)
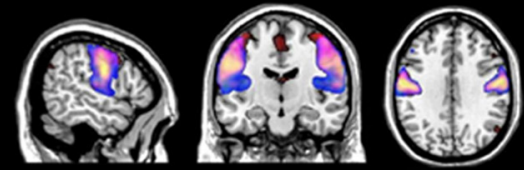

9. Medial temporal

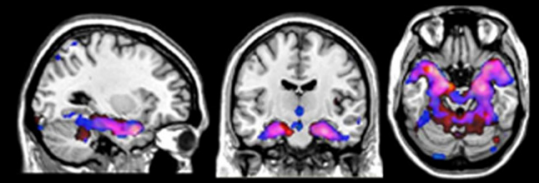

11. Ventral stream
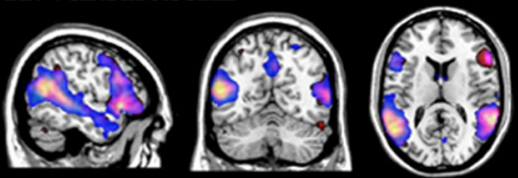

4. Auditory Network (AN)

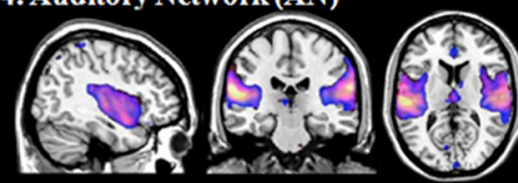

6. Default Mode Network (DMN)

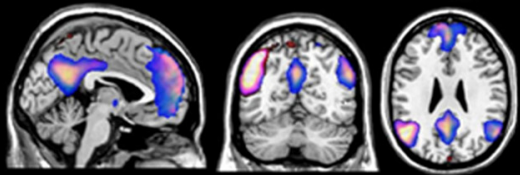

8. Sensorv Motor Network (SMN)

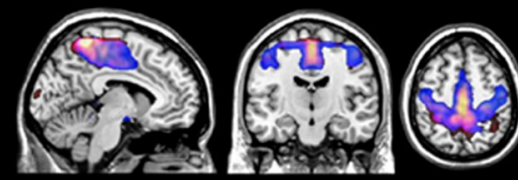

10. Task positive
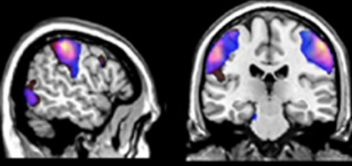

12. Salience network

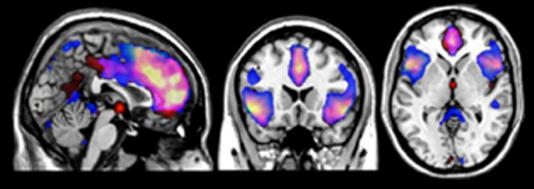

13. Left Frontoparietal Network (LFPN)
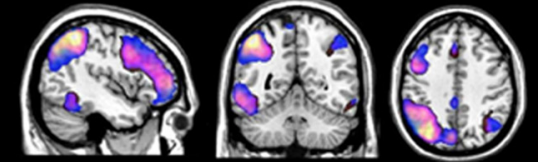

14. Right Frontoparietal Network (RFPN)
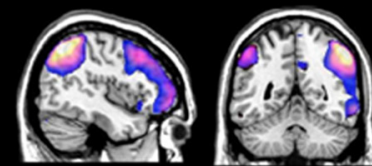

$L$

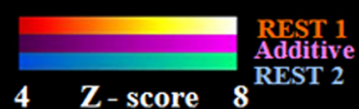

Figure 6. Group-ICA estimated RSN. Spatial maps of 14 ICs identified as RSNs of each rs-fMRI period: pretraining (red- orange bar), posttraining (blue-green bar), and the common regions (violet bar; overlaid on the MNI standard brain). The 14RSNs consist of three networks corresponding to the visual system represented by the (1) primary visual network (inferior occipital gyrus), (2) lateral visual network (middle occipital gyrus), and (3) medial visual network (superior occipital gyrus); (4) the auditory network (AN), which includes the bilateral middle and superior temporal gyri, posterior insular cortex, superior temporal sulcus, and Heschl gyrus; (5) precuneus network; (6) default mode network (DMN) involving the posterior cingulated cortex/precuneus region, bilateral inferior parietal gyrus, middle temporal gyrus, and anterior cingulate gyrus; two more networks corresponding to motor and somatosensory functions, the (7) sensory network and (8) sensory-motor network; (9) the medial temporal network including the hippocampus-amygdala complex; the attentional networks composed of the (10) task-positive network reminiscent of the dorsal attention network, (11) ventral stream network, and (12) salience network including the anterior cingulate, bilateral anterior insular, and dorsolateral prefrontal cortices; and (13) left frontoparietal (LFPN) and (14) right frontoparietal (RFPN) lateralized networks including the dorsolateral prefrontal cortex, ventrolateral prefrontal cortex, dorsomedial prefrontal cortex, and parietal cortices, as well as a site in the anterior insula. L, Left; $R$, right.

presented significant changes in rs-FC (LFO/aI and LSPL). Using this approach, we observed two distinct FC maps (Fig. 7A, pretraining, $B$, posttraining). Finally, we performed a spatial correlation of the two FC maps with each RSN obtained by ICA before
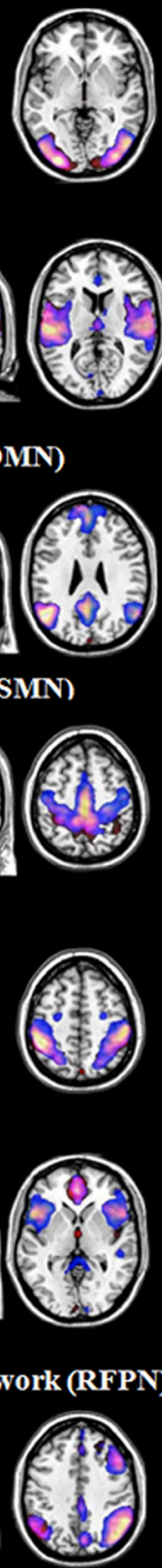

and after training to select the RSN associated with the LFO/aI and LSPL seeds (Fig. 7C). Considering the magnitude of these spatial correlations, and after confirming the correspondence between FC maps and RSNs through visual evaluation (Correa et al., 2007), we determined that the RSNs of interest associated with the LFO/aI were the salience network and the left frontoparietal network, whereas only the left frontoparietal network was associated with the LSPL.

We then calculated the intrinsic rs-FC of each seed within each associated RSN (Fig. 7D). Results showed that the intrinsic rs-FC of the LFO/aI decreased within the left frontoparietal network and increased within the salience network after learning, while the LSPL was similarly anchored in the left frontoparietal network before and after learning (Fig. 7D). Moreover, the intrinsic change in rs-FC of the LFO/aI within the salience network correlated negatively with posttraining rs-FC between the LFO/aI and LSPL (Fig. 7E). Therefore, less posttraining rs-FC between the LFO/aI and LSPL was associated with stronger involvement of the LFO/aI in the salience network after training.

\section{Experiment 2}

As in Experiment 1, results confirmed that performance improved with training. A one-way repeated-measures ANOVA, with time (Test 1 , Test 2 , and Test 3 ) as an independent variable, revealed a significant linear effect $\left(F_{(1,27)}=30.74, p<\right.$ 0.001 ), demonstrating a clear improvement in performance as a function of training (Fig. 8A).

Replicating Experiment 1, we found that the pretraining rs-FC between the LFO/aI and LSPL correlated significantly with posttraining performance (Test 3; Fig. $8 B$ ). We additionally performed a partial correlation analysis controlling for Tests 1 and 2 together and separately (Test $1, r=0.36, p=0.06$; Test 2, $r=0.46, p<$ $0.05)$.

\section{Discussion}

In the present study, we obtained converging evidence with past results demonstrating that the LSPL, LFO/aI, and LSMG increased in activation after learning and during processing of the nonnative phonetic contrast (Golestani and Zatorre, 2004). Crucially, the current results are the first to our knowledge to demonstrate that initial rs-FC between areas related to learning a nonnative phonetic contrast (i.e., the LFO/aI and LSPL) could account for the degree of learning ability after distributed (Experiment 1) and intensive (Experiment 2) training. 
A

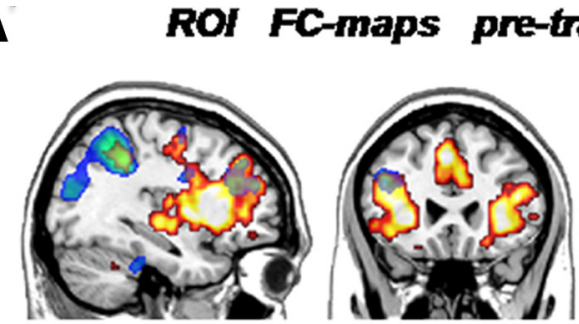

B ROI FC-maps post-training

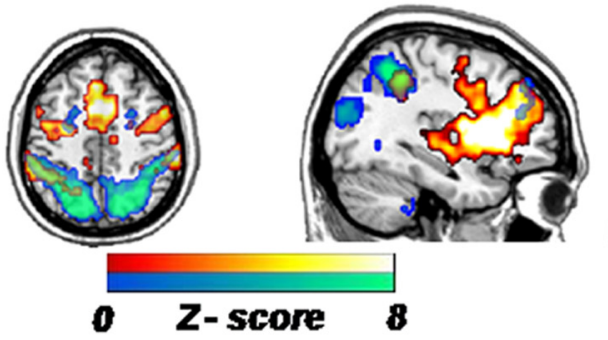

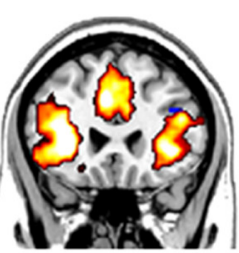

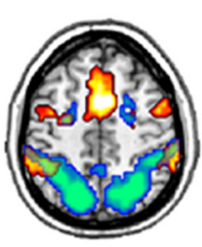

C

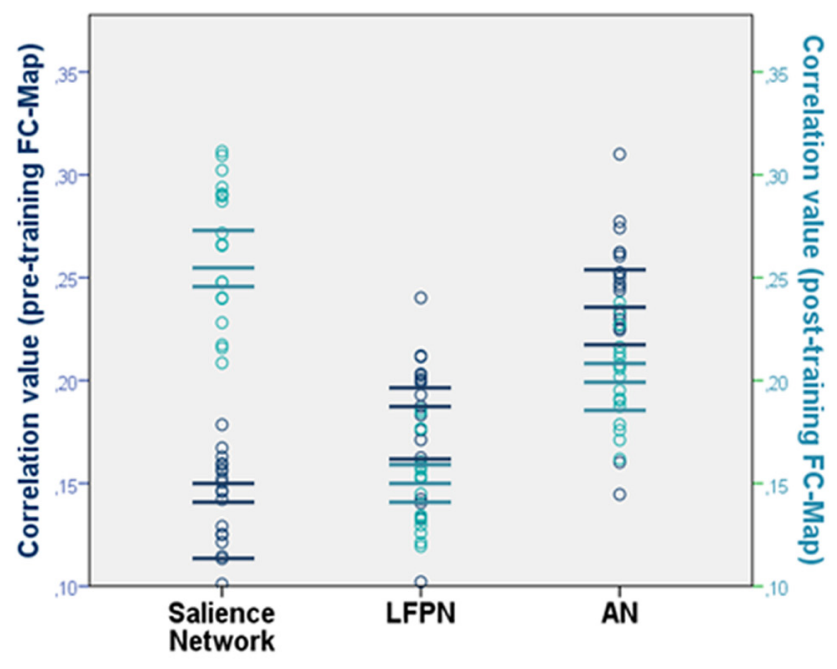

D

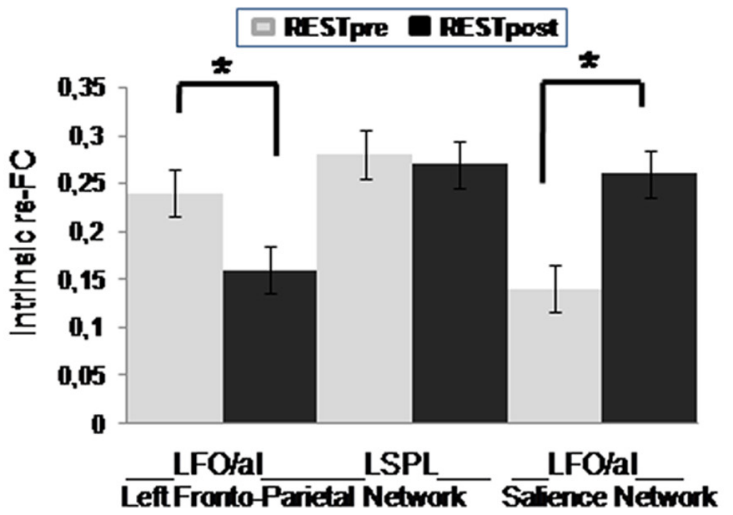

LSPL FC-Map

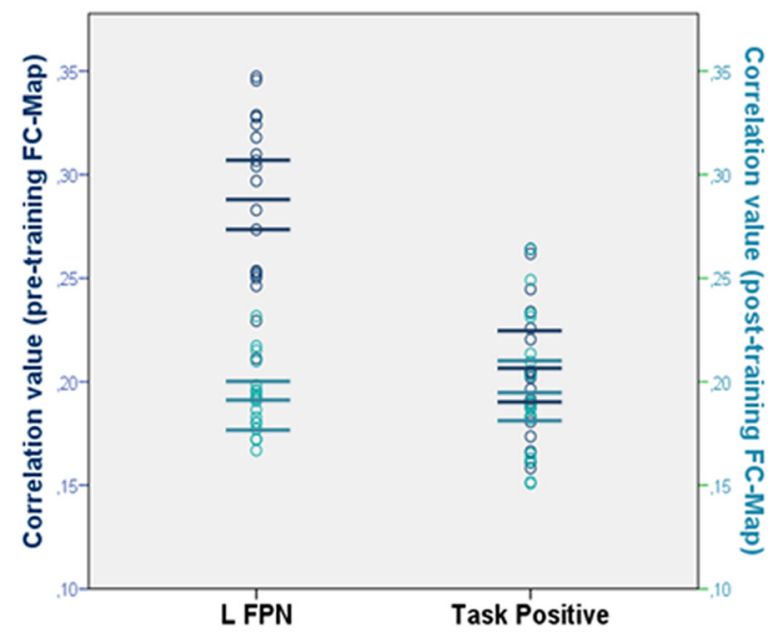

E

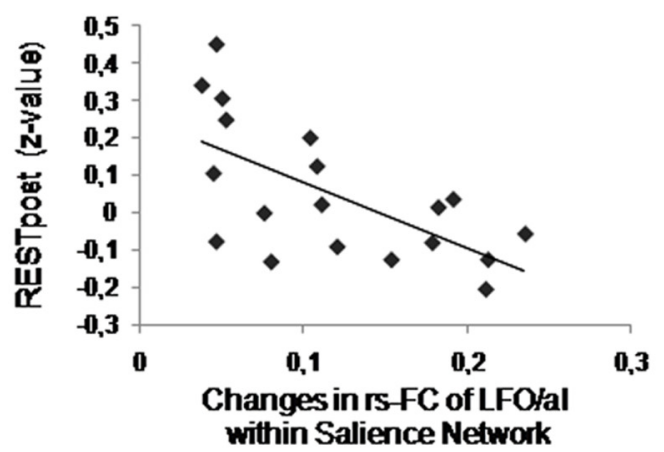

Figure 7. Networks during rs-fMRI periods. $A, B$, Temporal correlations in BOLD signal for each seed ( $p<0.05$, FWE cluster corrected) determined the FC map of the LFO/al (red- orange bar) and LPSL (blue-green bar) before training $(\boldsymbol{A})$ and after training $(\boldsymbol{B})$. $\boldsymbol{A}$, Before training, the LF0/al showed rs-FC with the right inferior frontal operculum/anterior insula, bilateral frontal lobe, bilateral temporal lobe, cingulate gyrus, bilateral inferior parietal lobe, bilateral caudate, thalamus, and LSPL. On the other hand, the LSPL involved the bilateral inferior and superior parietal lobe, bilateral inferior temporal gyrus, bilateral middle frontal gyrus, bilateral superior frontal gyrus, cingulate gyrus, right inferior frontal gyrus, and left frontal operculum. $\boldsymbol{B}$, After training, the LFO/al showed rs- $\mathrm{FC}$ with the same areas as before training except with the LSPL, and the LSPL also showed rs- $\mathrm{FC}$ with the same areas as before training except with the left frontal operculum. $C$, Individual variance in correlation using these $\mathrm{FC}$ maps as templates for a subsequent ICA. Spatial correlation scores obtained through the spatial correlation sorting option in GIFT are shown for each participant's best-fit images. The salience network, left frontoparietal network (LFPN), and auditory network (AN) were associated with the LFO/al FC map, and the LFPN and task-positive network were associated with the LSPL FC map. Bars indicate means and 95\% confidence intervals. D, The weighted mean of each seed within the RSNs, obtained by extracting the first eigenvariate in SPM8 and representing intrinsic rs- $\mathrm{FC}$, was used for paired $t$ test analyses. The results demonstrated a significant increase in intrinsic rs- $F$ C of the LFO/al within the salience network $\left(t_{(18)}=7.723\right)$ and a significant decrease in intrinsic rs-FC within the left frontoparietal network $\left(t_{(18)}=-3.89\right)$ after training $\left({ }^{*} p<0.001\right)$, while no change was observed for the LSPL within the left frontoparietal network. $E$, Changes in intrinsic rs-FC of the LF0/al within the salience network correlated negatively with posttraining rs-FC of the LF0/al and LSPL $\left(r_{s}=-0.59, p<0.01, n=19\right)$. L, Left; R, right.

Furthermore, Experiment 1 also described how the intrinsic activity of the LFO/aI within its associated RSN was modified after 2 weeks of training in phonetic learning. Thus, rs-FC between brain areas involved in phonetic learning may predict learning outcomes before experience comes into play, and may serve to explain how the brain is modified by learning.
Participants in both experiments showed poor performance identifying the Hindi nonnative contrast at baseline, but considerable improvement whether they had 2 weeks or a single hour of intense training. Experiment 1 demonstrated that distributed training was associated with increased activation in languagerelated areas such as the LFO/aI and LSMG (Golestani and Za- 


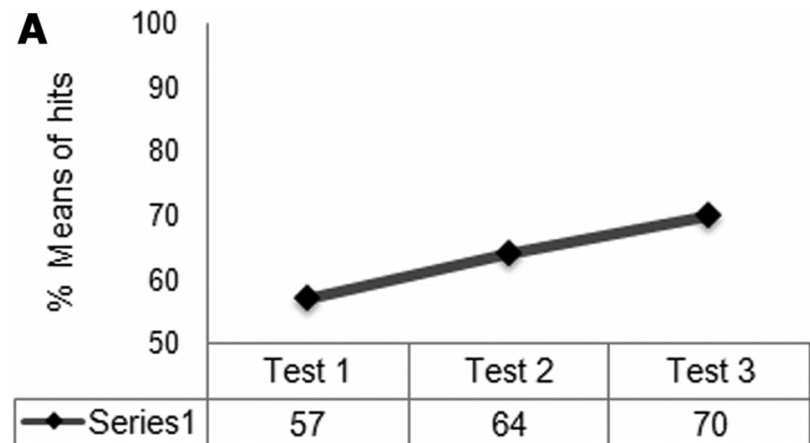

B

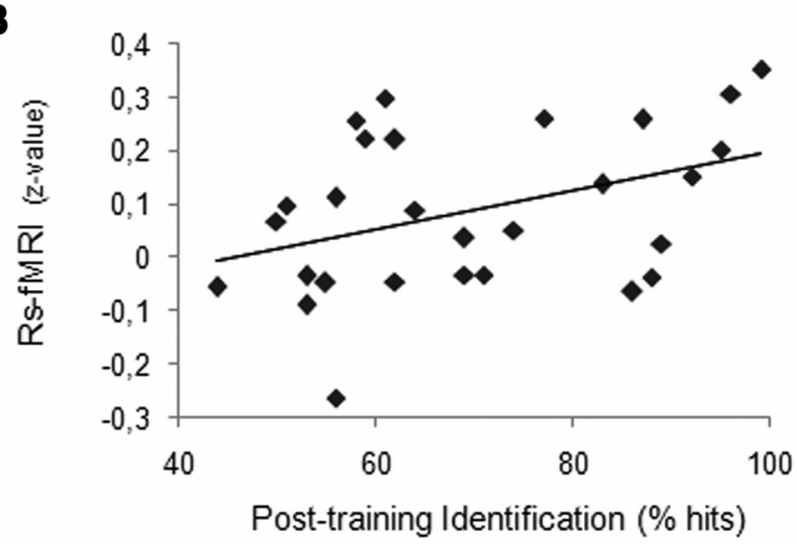

Figure 8. Changes in rs- $\mathrm{FC}$ associated with nonnative phoneme identification training in Experiment 2. A, Behavioral performance of 28 participants on the identification of the nonnative contrast. The three learning measures after each training session (learning curve) showed that performance improved with training. $\boldsymbol{B}$, rs-FC between the LFO/al and LSPL of 28 participants became significantly correlated with Test 3 (posttraining identification performance; $\left.r_{s}=0.41, p<0.05\right)$, replicating the results obtained in Experiment 1.

torre, 2004). These language-related areas participate in different language functions, including articulatory planning and covert articulation (Brown et al., 2009; Price, 2010). Importantly, our results also showed that activation in the LFO/aI correlated positively with task performance. The LSPL increased in activation with learning as well, although this brain area is not directly related to language. However, Golestani and Zatorre (2004) also reported activation in the LSPL after phonetic learning. In addition, another previous study of this group found a relationship between increased white volume in the left inferior and superior parietal areas and faster learning of a nonnative contrast (Golestani et al., 2002). Finally, previous results have shown that the LSPL might contribute to auditory selective attention in complex situations (Bishop and Miller, 2009; Westerhausen et al., 2010).

The pattern of rs-FC and RSNs obtained in our resting-state analyses also confirmed previous results. First, the pattern of rs-FC between task-related seeds was consistent with previous descriptions of brain networks. More concretely, the pattern of connectivity between the relevant regions described previously was consistent with a previous study of 970 healthy participants, designed to describe the language networks (Tomasi and Volkow, 2012). First, we found strong rs-FC between LFO/aI and LSMG, two brain areas included in the language networks. Second, and importantly for this study, weak rs-FC between LFO/aI and LSPL was also found in Tomasi and Volkow's (2012) study. Third, activity in the LMTG (in this study, related to native phoneme identification) has been associated previously with the auditory network. In addition, the LMTG has been shown to be poorly correlated or anticorrelated with other language areas (LFO/aI and LSMG), reflecting functional segregation of the auditory cortex and language areas. Finally, the strong correlation between the LSPL and LSMG reflected the adscription of both areas to the left frontoparietal network (Nelson et al., 2010).

The second aspect in resting-state analyses is the identification of RSNs according to previous literature (Fig. 6) (Veer et al., 2010; Allen et al., 2011). Importantly for the present study, ICA has revealed the involvement of both the left frontoparietal and salience networks both at pretraining and posttraining, and we have identified the LFO/aI as a key node common to both networks. The left frontoparietal network is thought to mediate goal-directed top-down processing (Corbetta and Shulman, 2002; Vincent et al., 2008). In the specific case of auditory processing, this network is activated in situations requiring active top-down processing of complex auditory information (Westerhausen et al., 2010) as well as bottom-up triggered and top-down controlled shifting between auditory stimuli (Salmi et al., 2009). The salience network is a task-control network related to the resolution of conflicts and ambiguities, especially to stimuli with a certain degree of personal salience (Ridderinkhof et al., 2004; Klein et al., 2007; Eichele et al., 2008).

Considering the replication of previous task-related fMRI and resting-state results, the key finding of both experiments in the present study is that the rs-FC between the LSPL and LFO/aI represents a neural predictor of learning outcomes after training. In other words, we can relate nonnative contrast learning to a prelearning measure of rs-FC between brain areas involved in the task. This crucial finding should be interpreted in light of further results obtained in the present study. First, the mean magnitude of this correlation was rather low. Second, the rs-FC between these areas presented an important degree of variability across participants (from $r=-0.26$ to $r=0.56$ ), especially compared to the strong rs-FC between the LSMG and LFO/aI and between the LSMG and LSPL. This variability may reflect the possible existence of a dorsal component of the inferior frontal occipital fasciculus that connects the superior parietal lobe and frontal operculum, described in a reduced number of participants (i.e., 64\%) in recent postmortem studies (Martino et al., 2010, 2011). We may then speculate that the degree of coherence in rs-FC may be associated with individual differences in structural connectivity between the LSPL and LFO/aI that may facilitate subsequent auditory discrimination of complex sounds.

A third relevant factor was that the only significant change in rs-FC between seeds after training was observed between the LFO/aI and LSPL; that is, training significantly reduced the mean connectivity of spontaneous brain activity between these areas, and the magnitude of this reduction was positively correlated with learning outcomes. Studying the RSNs associated with these seeds revealed that learning caused activity of the LFO/aI to be decoupled from the left frontoparietal network and increased intrinsic activity within the salience network. In this sense, the LFO/aI seemed to operate to identify new salient stimuli through the salience network. After 2 weeks of learning and receiving continuous feedback during phonemic training, these stimuli became salient (participants were able to discriminate them), the task-evoked brain activity of the LSPL and LFO/aI increased considerably in the presence of these stimuli, and the LFO/aI modified its intrinsic brain activity at rest (more related to the salience network). In sum, nonnative identification learning over 2 weeks had probably sculpted brain activity in the area most related to phonemic learning by biasing its activity toward the management of salient stimuli. Future studies should confirm the relevance of 
this mechanism in other language tasks as well as the persistence of these changes in the brain.

Finally, Experiment 2 was designed to confirm the crucial result obtained in Experiment 1 in a new and larger sample. Results corroborated that pretraining rs-FC between the LSPL and $\mathrm{LFO} / \mathrm{aI}$ predicted the ability for phonetic learning. Although the training was intensive $(1 \mathrm{~h})$ in this experiment, the rs-FC between target seeds was similarly correlated with final performance and learning. Future studies should elucidate if the changes in brain task activity and networks reported in Experiment 1 would be observed after this intensive training.

We conclude that our findings demonstrate the capacity of rs-fMRI not only to predict learning outcomes, but also to determine brain changes associated with learning by analyzing changes in rs-FC. Previous results have shown that spontaneous activity in the brain measured by rs-fMRI may be related to actual anatomical circuitry, cognitive performance, and behavioral deficits (Baldassarre et al., 2012). Our results unveil that the spontaneous coherence in the brain may also reflect its potential to incorporate new knowledge. Furthermore, our longitudinal analysis combining both task-related fMRI and rs-fMRI has allowed us to establish a correspondence between different brain regions involved in learning and to use spontaneous activity in these specific areas to account for individual differences in learning. Generalizing the specific methodology used here may serve to determine a priori the potentialities of the brain with subsequent applicability in the fields of education and clinical health.

\section{Notes}

Supplemental material for this article is available at www.fmri.uji. es/data/SI_Ventura-CamposN.doc. Supplemental Table 1 shows a onesample $t$ test of the phoneme identification fMRI task, and this table is related to Figure 3. Supplemental Table 2 shows comparison and correlation analysis of the phoneme identification fMRI task for the nonnative contrast and is related to Figure 4, $A$ and $B$. Supplemental Table 3 shows seed regions for rs-FC analysis. Supplemental Table 4 shows spatial correlation and IC sorting using FC maps as templates and is related to Figure 7C. This material has not been peer reviewed.

\section{References}

Abou-Elseoud A, Starck T, Remes J, Nikkinen J, Tervonen O, Kiviniemi V (2010) The effect of model order selection in group PICA. Hum Brain Mapp 31:1207-1216. Medline

Allen EA, Erhardt EB, Damaraju E, Gruner W, Segall JM, Silva RF, Havlicek M, Rachakonda S, Fries J, Kalyanam R, Michael AM, Caprihan A, Turner JA, Eichele T, Adelsheim S, Bryan AD, Bustillo J, Clark VP, Feldstein Ewing SW, Filbey F, et al. (2011) A baseline for the multivariate comparison of resting-state networks. Front Syst Neurosci 5:2. Medline

Baldassarre A, Lewis CM, Committeri G, Snyder AZ, Romani GL, Corbetta M (2012) Individual variability in functional connectivity predicts performance of a perceptual task. Proc Natl Acad Sci U S A 109:3516-3521. CrossRef Medline

Beckmann CF, DeLuca M, Devlin JT, Smith SM (2005) Investigations into resting-state connectivity using independent component analysis. Philos Trans R Soc Lond B Biol Sci 360:1001-1013. CrossRef Medline

Bell AJ, Sejnowski TJ (1995) An information-maximization approach to blind separation and blind deconvolution. Neural Comput 7:1129-1159. CrossRef Medline

Bishop CW, Miller LM (2009) A multisensory cortical network for understanding speech in noise. J Cogn Neurosci 21:1790-1805. CrossRef Medline

Biswal B, Yetkin FZ, Haughton VM, Hyde JS (1995) Functional connectivity in the motor cortex of resting human brain using echo-planar MRI. Magn Reson Med 34:537-541. CrossRef Medline

Brown S, Laird AR, Pfordresher PQ, Thelen SM, Turkeltaub P, Liotti M (2009) The somatotopy of speech: phonation and articulation in the human motor cortex. Brain Cogn 70:31-41. CrossRef Medline

Buckner RL, Andrews-Hanna JR, Schacter DL (2008) The brain's default network: anatomy, function, and relevance to disease. Ann N Y Acad Sci 1124:1-38. CrossRef

Calhoun VD, Adali T, Pearlson GD, Pekar JJ (2001) A method for making group inferences from functional MRI data using independent component analysis. Hum Brain Mapp 14:140-151. CrossRef Medline

Calhoun VD, Pekar JJ, McGinty VB, Adali T, Watson TD, Pearlson GD (2002) Different activation dynamics in multiple neural systems during simulated driving. Hum Brain Mapp 16:158-167. CrossRef Medline

Calhoun VD, Kiehl KA, Pearlson GD (2008) Modulation of temporally coherent brain networks estimated using ICA at rest and during cognitive tasks. Hum Brain Mapp 29:828-838. CrossRef Medline

Chao-Gan Y, Yu-Feng Z (2010) DPARSF: a MATLAB toolbox for "pipeline" data analysis of resting-state fMRI. Front Syst Neurosci 4:13. Medline

Cole DM, Smith SM, Beckmann CF (2010) Advances and pitfalls in the analysis and interpretation of resting-state FMRI data. Front Syst Neurosci 4:8. Medline

Corbetta M, Shulman GL (2002) Control of goal-directed and stimulusdriven attention in the brain. Nat Rev Neurosci 3:201-215. Medline

Correa N, Adali T, Calhoun VD (2007) Performance of blind source separation algorithms for fMRI analysis using a group ICA method. Magn Reson Imaging 25:684-694. CrossRef Medline

Damoiseaux JS, Rombouts SA, Barkhof F, Scheltens P, Stam CJ, Smith SM, Beckmann CF (2006) Consistent resting-state networks across healthy subjects. Proc Natl Acad Sci U S A 103:13848-13853. CrossRef Medline

Deng Y, Booth JR, Chou TL, Ding GS, Peng DL (2008) Item-specific and generalization effects on brain activation when learning Chinese characters. Neuropsychologia 46:1864-1876. CrossRef Medline

Eichele T, Debener S, Calhoun VD, Specht K, Engel AK, Hugdahl K, von Cramon DY, Ullsperger M (2008) Prediction of human errors by maladaptive changes in event-related brain networks. Proc Natl Acad Sci U S A 105:6173-6178. CrossRef Medline

Erhardt EB, Rachakonda S, Bedrick EJ, Allen EA, Adali T, Calhoun VD (2011) Comparison of multi-subject ICA methods for analysis of fMRI data. Hum Brain Mapp 32:2075-2095. CrossRef Medline

Fox MD, Snyder AZ, Vincent JL, Corbetta M, Van Essen DC, Raichle ME (2005) The human brain is intrinsically organized into dynamic, anticorrelated functional networks. Proc Natl Acad Sci U S A 102:9673-9678. CrossRef Medline

Friston KJ, Rotshtein P, Geng JJ, Sterzer P, Henson RN (2006) A critique of functional localisers. Neuroimage 30:1077-1087. CrossRef Medline

Golestani N, Zatorre RJ (2004) Learning new sounds of speech: reallocation of neural substrates. Neuroimage 21:494-506. CrossRef Medline

Golestani N, Zatorre RJ (2009) Individual differences in the acquisition of second language phonology. Brain Lang 109:55-67. CrossRef Medline

Golestani N, Paus T, Zatorre RJ (2002) Anatomical correlates of learning novel speech sounds. Neuron 35:997-1010. CrossRef Medline

Himberg J, Hyvärinen A, Esposito F (2004) Validating the independent components of neuroimaging time series via clustering and visualization. Neuroimage 22:1214-1222. CrossRef Medline

Kiviniemi V, Starck T, Remes J, Long X, Nikkinen J, Haapea M, Veijola J, Moilanen I, Isohanni M, Zang YF, Tervonen O (2009) Functional segmentation of the brain cortex using high model order group PICA. Hum Brain Mapp 30:3865-3886. CrossRef Medline

Klatt D (1980) Software for a cascade/parallel formant synthesizer. J Acoust Soc Am 67:13-33.

Klein TA, Endrass T, Kathmann N, Neumann J, von Cramon DY, Ullsperger M (2007) Neural correlates of error awareness. Neuroimage 34:17741781. CrossRef Medline

Lewis CM, Baldassarre A, Committeri G, Romani GL, Corbetta M (2009) Learning sculpts the spontaneous activity of the resting human brain. Proc Natl Acad Sci U S A 106:17558-17563. CrossRef Medline

Lowe MJ, Mock BJ, Sorenson JA (1998) Functional connectivity in single and multislice echoplanar imaging using resting-state fluctuations. Neuroimage 7:119-132. CrossRef Medline

Martino J, Brogna C, Robles SG, Vergani F, Duffau H (2010) Anatomic dissection of the inferior fronto-occipital fasciculus revisited in the lights of brain stimulation data. Cortex 46:691-699. CrossRef Medline

Martino J, De Witt Hamer PC, Vergani F, Brogna C, de Lucas EM, VázquezBarquero A, García-Porrero JA, Duffau H (2011) Cortex-sparing fiber dissection: an improved method for the study of white matter anatomy in the human brain. J Anat 219:531-541. CrossRef Medline 
Nelson SM, Cohen AL, Power JD, Wig GS, Miezin FM, Wheeler ME, Velanova K, Donaldson DI, Phillips JS, Schlaggar BL, Petersen SE (2010) A parcellation scheme for human left lateral parietal cortex. Neuron 67: 156-170. CrossRef Medline

Poldrack RA (2000) Imaging brain plasticity: conceptual and methodological issues-a theoretical review. Neuroimage 12:1-13. CrossRef Medline

Polka L (1991) Cross-language speech perception in adults: phonemic, phonetic, and acoustic contributions. J Acoust Soc Am 89:2961-2977. CrossRef Medline

Price CJ (2010) The anatomy of language: a review of 100 fMRI studies published in 2009. Ann N Y Acad Sci 1191:62-88. CrossRef

Ridderinkhof KR, Ullsperger M, Crone EA, Nieuwenhuis S (2004) The role of the medial frontal cortex in cognitive control. Science 306:443-447. CrossRef Medline

Salmi J, Rinne T, Koistinen S, Salonen O, Alho K (2009) Brain networks of bottom-up triggered and top-down controlled shifting of auditory attention. Brain Res 1286:155-164. CrossRef Medline

Seeley WW, Menon V, Schatzberg AF, Keller J, Glover GH, Kenna H, Reiss AL, Greicius MD (2007) Dissociable intrinsic connectivity networks for salience processing and executive control. J Neurosci 27:2349-2356. CrossRef Medline

Shehzad Z, Kelly AM, Reiss PT, Gee DG, Gotimer K, Uddin LQ, Lee SH, Margulies DS, Roy AK, Biswal BB, Petkova E, Castellanos FX, Milham MP (2009) The resting brain: unconstrained yet reliable. Cereb Cortex 19: 2209-2229. CrossRef Medline

Smith SM, Fox PT, Miller KL, Glahn DC, Fox PM, Mackay CE, Filippini N, Watkins KE, Toro R, Laird AR, Beckmann CF (2009) Correspondence of the brain's functional architecture during activation and rest. Proc Natl Acad Sci U S A 106:13040-13045. CrossRef Medline

Staeren N, Renvall H, De Martino F, Goebel R, Formisano E (2009) Sound categories are represented as distributed patterns in the human auditory cortex. Curr Biol 19:498-502. CrossRef Medline

Takeuchi H, Taki Y, Hashizume H, Sassa Y, Nagase T, Nouchi R, Kawashima $\mathrm{R}$ (2011) Effects of training of processing speed on neural systems. J Neurosci 31:12139-12148. CrossRef Medline

Tomasi D, Volkow ND (2012) Resting functional connectivity of language networks: characterization and reproducibility. Mol Psychiatry 17:841854. CrossRef Medline

Veer IM, Beckmann CF, van Tol MJ, Ferrarini L, Milles J, Veltman DJ, Aleman A, van Buchem MA, van der Wee NJ, Rombouts SA (2010) Whole brain resting-state analysis reveals decreased functional connectivity in major depression. Front Syst Neurosci 4:41

Vincent JL, Kahn I, Snyder AZ, Raichle ME, Buckner RL (2008) Evidence for a frontoparietal control system revealed by intrinsic functional connectivity. J Neurophysiol 100:3328-3342. CrossRef Medline

Werker JF, Lalonde CE (1988) Cross-language speech perception:initial capabilities and development change. Dev Psychobiol 24:672-683. CrossRef

Westerhausen R, Moosmann M, Alho K, Belsby SO, Hämäläinen H, Medvedev S, Specht K, Hugdahl K (2010) Identification of attention and cognitive control networks in a parametric auditory fMRI study. Neuropsychologia 48:2075-2081. CrossRef Medline

Ystad M, Eichele T, Lundervold AJ, Lundervold A (2010) Subcortical functional connectivity and verbal episodic memory in healthy elderly-a resting state fMRI study. Neuroimage 52:379-388. CrossRef Medline 